\title{
An IGRF candidate main geomagnetic field model for epoch 2000 and a secular variation model for 2000-2005
}

\author{
Benoit Langlais and Mioara Mandea \\ Institut de Physique du Globe de Paris, B.P. 89, 4 Place Jussieu, 75252 Paris cedex 5, France
}

(Received February 14, 2000; Revised July 21, 2000; Accepted July 21, 2000)

\begin{abstract}
A candidate main geomagnetic field model for epoch 2000, and a secular variation model for the period 20002005 , are proposed. The main field model is to degree and order 10, the secular variation one to degree and order 8 . These models are derived using the method of least squares. A 1997.5 main field model was derived from annual mean values provided by geomagnetic observatories for the 1997.5 epoch, repeat station measurements made in 1997 and reduced to 1997.5 , and scalar data since 1995 adjusted to 1997.5. A weighting scheme based on both geographical distribution and data quality was applied. This model was then extrapolated to the 2000.0 epoch, using previously derived secular variation models. To derive these secular variation models, twenty six main field models were firstly computed for epochs 1975.5 through 2000.5, using annual mean values of the $X, Y, Z$ components of the magnetic field from observatories, with the same geographical distribution every year. When missing, annual mean values for 1998, 1999 and 2000 were estimated from extrapolated monthly means, using exponential smoothing and taking account of the seasonal variation. From these twenty six models, twenty five annual secular variation models were extracted, by taking the differences between consecutive main field models. Finally, to produce the IGRF candidate secular variation model, each Gauss coefficient of this set of secular variation models was extrapolated to give values for each year to 2005, using exponential smoothing. So, a mean secular variation model was obtained for the period 2000-2005 and this is proposed for adoption.
\end{abstract}

\section{Introduction}

The temporal variation of the geomagnetic field covers a large range of time-scales, from seconds to millennia. Accurate models of the geomagnetic main field and its secular variation are very important for the evaluation of the temporal changes (with time constants of order of a year to a century), but also for many other studies, such as the physics of the Earth's deep interior, global or regional mapping.

To derive good models, data well distributed all over the globe and over long periods of time are essential. The best geographical distribution is provided by satellite data. Unfortunately, up to now there have been few satellite missions dedicated to measuring the geomagnetic field, and only two vectorial satellites. After the launch of the MAGSAT satellite (1979), twenty years have passed until the launch of the new vectorial satellite, Ørsted (February, 23rd 1999)_over this time-span the only other important satellite mission was POGS, which measured only total field intensity with poor accuracy in 1993-1994.

Modelling the main field and its secular variation over long periods of time depends greatly on the network of geomagnetic observatories, which record continuously three components of the field. The present-day network is far from an ideal one, and it needs to be extended and improved, even if in the future satellites measure the field continuously.

It is common to present the internal magnetic field of the Earth in terms of a potential function $V_{i}(r, \theta, \phi)$, which can

Copy right(c) The Society of Geomagnetism and Earth, Planetary and Space Sciences (SGEPSS); The Seismological Society of Japan; The Volcanological Society of Japan; The Geodetic Society of Japan; The Japanese Society for Planetary Sciences. be expressed as a spherical harmonic expansion:

$$
\begin{aligned}
V_{i}(r, \theta, \phi)= & a \sum_{n=1}^{N} \sum_{m=0}^{n}\left(\frac{a}{r}\right)^{n+1}\left(g_{n}^{m} \cos m \phi\right. \\
& \left.+h_{n}^{m} \sin m \phi\right) P_{n}^{m}(\cos \theta)
\end{aligned}
$$

where $a(6371.2 \mathrm{~km})$ is the mean radius of the Earth, $r$ denotes the radial distance from the center of the Earth, $\theta$ and $\phi$ denote the geocentric colatitude and longitude of a given point. $P_{n}^{m}(\cos \theta)$ denote the Schmidt-normalized associated Legendre functions of degree $n$ and order $m$, and $g_{n}^{m}$ and $h_{n}^{m}$ denote the Gauss coefficients. Their temporal dependence is:

$$
\begin{aligned}
& g_{n}^{m}(t)=g_{n}^{m}\left(T_{0}\right)+g_{n}^{m}\left(t-T_{0}\right) \\
& h_{n}^{m}(t)=h_{n}^{m}\left(T_{0}\right)+\dot{h}_{n}^{m}\left(t-T_{0}\right)
\end{aligned}
$$

where $g_{n}^{m}$ and $\dot{h_{n}^{m}}$ represent the Gauss coefficients for the secular variation, i.e., the first temporal derivative of $g_{n}^{m}$ and $h_{n}^{m}, T_{0}$ denotes the epoch of the main field model, and $t$ is the required epoch $\left(T_{0} \leq t \leq T_{0}+5\right)$.

It is now generally agreed that, near the Earth's surface, the magnetic field from the core dominates for the degrees 1-12 and that the magnetic field from the lithosphere dominates for degrees above 14 .

In our attempt to produce a candidate main field model for 2000 and a secular variation one for 2000-2005 we chose to concentrate as much as possible on observations made after 1995, the epoch for which the last IGRF model was derived. Unfortunately, at the time of computation, no Ørsted satellite 
Table 1. Observatories considered in the MF and SV modelling.

\begin{tabular}{|c|c|c|c|c|c|c|c|}
\hline Code $^{a}$ & $\lambda^{b}$ & $\varphi^{c}$ & Date $^{d}$ & $X_{c c}(\mathrm{nT})^{e}$ & $Y_{c c}(\mathrm{nT})^{f}$ & $Z_{c c}(\mathrm{nT})^{g}$ & Time period $^{h}$ \\
\hline AAA & 43.250 & 76.916 & 1980.0 & 158 & 14 & -8 & $1980.00-1998.83$ \\
\hline $\mathrm{AAE}$ & 9.000 & 38.800 & 1980.0 & 554 & -122 & 636 & $*$ \\
\hline $\mathrm{ABG}$ & 18.633 & 72.867 & 1980.0 & -35 & 476 & 659 & 1980.00-1997.92 \\
\hline $\mathrm{ABK}$ & 68.360 & 18.820 & 1980.0 & -10 & 69 & 34 & $*$ \\
\hline AIA & -65.250 & 295.733 & 1980.0 & 77 & -123 & 479 & $1980.00-1988.75$ \\
\hline ALE & 82.500 & 297.650 & 1980.0 & -29 & 73 & -102 & 1984.00-1997.92 \\
\hline AMS & -37.833 & 77.574 & 1982.5 & -643 & -413 & -2341 & $*$ \\
\hline API & -13.800 & 188.233 & 1980.0 & -54 & 199 & -914 & 1980.00-1997.92 \\
\hline AQU & 42.383 & 13.317 & 1980.0 & -18 & 45 & 36 & 1980.00-1997.92 \\
\hline ARS & 56.433 & 58.566 & 1980.0 & 77 & -196 & 528 & 1980.00-1998.92 \\
\hline ASC & -7.950 & 345.617 & 1993.5 & $\cdots$ & $\ldots$ & $\cdots$ & $*$ \\
\hline ASP & -23.767 & 133.883 & 1993.5 & $\cdots$ & $\cdots$ & $\cdots$ & $*$ \\
\hline $\mathrm{BDV}$ & 49.080 & 14.020 & 1980.0 & -52 & -13 & 2 & 1980.00-1998.67 \\
\hline BEL & 51.833 & 20.792 & 1980.0 & 106 & 125 & 336 & 1980.00-1998.92 \\
\hline BFE & 55.633 & 11.666 & 1980.0 & 50 & -79 & -159 & $1980.00-1998.42$ \\
\hline BJI & 40.033 & 116.183 & 1980.0 & 586 & -211 & 455 & 1980.00-1997.92 \\
\hline BLC & 64.317 & 263.983 & 1980.0 & 115 & -41 & -112 & 1980.00-1997.92 \\
\hline BNG & 4.433 & 18.566 & 1980.0 & -187 & -36 & 290 & $1980.00-1998.58$ \\
\hline $\mathrm{BOU}$ & 40.140 & 254.766 & 1980.0 & -30 & 15 & -137 & 1980.00-1997.92 \\
\hline BOX & 58.033 & 38.966 & 1980.0 & -22 & -46 & -405 & $*$ \\
\hline BRW & 71.323 & 203.380 & 1980.0 & -14 & -78 & -3 & 1980.00-1997.92 \\
\hline BSL & 30.400 & 270.600 & 1987.5 & $\cdots$ & $\cdots$ & $\cdots$ & $*$ \\
\hline $\mathrm{CBB}$ & 69.117 & 254.967 & 1980.0 & 44 & -105 & 28 & 1980.00-1997.92 \\
\hline CBI & 27.100 & 142.183 & 1980.0 & -328 & -27 & 218 & $*$ \\
\hline $\mathrm{CCS}$ & 77.717 & 104.283 & 1980.0 & -76 & -103 & -57 & $*$ \\
\hline CHD & 31.000 & 103.700 & 1982.5 & -13 & 71 & -114 & $*$ \\
\hline CLF & 48.016 & 2.266 & 1980.0 & -89 & 2 & 132 & 1980.00-1998.92 \\
\hline $\mathrm{CMO}$ & 64.870 & 213.856 & 1980.0 & 10 & -62 & 23 & 1980.00-1997.92 \\
\hline $\mathrm{CNB}$ & -35.317 & 149.367 & 1980.0 & 30 & 46 & 117 & $1980.00-1998.83$ \\
\hline $\mathrm{CNH}$ & 43.827 & 125.299 & 1980.0 & -129 & 33 & 192 & 1980.00-1997.92 \\
\hline $\mathrm{COI}$ & 40.216 & 351.583 & 1980.0 & 37 & -19 & 8 & $1980.00-1998.83$ \\
\hline $\mathrm{CRP}$ & 10.433 & 275.083 & 1979.9 & -218 & -105 & -514 & $*$ \\
\hline CSY & -66.283 & 110.533 & 1980.0 & 844 & -264 & -842 & $*$ \\
\hline CTA & -20.083 & 146.250 & 1984.5 & -585 & -107 & 141 & $*$ \\
\hline $\mathrm{CZT}$ & -46.433 & 51.866 & 1980.0 & -792 & 833 & -111 & 1980.00-1997.92 \\
\hline DIK & 73.543 & 80.562 & 1980.0 & -279 & -197 & -144 & $*$ \\
\hline DLR & 29.490 & 259.083 & 1983.5 & 88 & 23 & 86 & $*$ \\
\hline DOB & 62.066 & 9.116 & 1980.0 & -106 & -54 & -232 & 1980.00-1998.50 \\
\hline DOU & 50.100 & 4.066 & 1980.0 & -3 & 80 & 118 & 1980.00-1998.92 \\
\hline DRV & -66.666 & 140.016 & 1980.0 & -136 & -394 & -2827 & 1980.00-1997.92 \\
\hline EBR & 40.816 & 0.500 & 1980.0 & 41 & 21 & -32 & $*$ \\
\hline ESK & 55.316 & 356.800 & 1980.0 & -15 & -27 & -59 & 1980.00-1998.92 \\
\hline ETT & 9.167 & 78.017 & 1980.5 & 98 & -45 & 95 & $*$ \\
\hline EYR & -43.416 & 172.350 & 1980.0 & -26 & -27 & 39 & $1980.00-1998.92$ \\
\hline FCC & 58.767 & 265.917 & 1980.0 & -155 & 30 & -248 & 1980.00-1997.92 \\
\hline FRD & 38.210 & 282.633 & 1980.0 & 34 & -79 & 105 & $1980.00-1997.92$ \\
\hline FRN & 37.090 & 240.280 & 1983.5 & -51 & -67 & -110 & $*$ \\
\hline
\end{tabular}


Table 1. (continued).

\begin{tabular}{|c|c|c|c|c|c|c|c|}
\hline Code $^{a}$ & $\lambda^{b}$ & $\varphi^{c}$ & Date $^{d}$ & $X_{c c}(\mathrm{nT})^{e}$ & $Y_{c c}(\mathrm{nT})^{f}$ & $Z_{c c}(\mathrm{nT})^{g}$ & Time period ${ }^{h}$ \\
\hline FUQ & 5.467 & 286.267 & 1980.0 & 81 & -83 & 50 & $1980.00-1997.50$ \\
\hline FUR & 48.166 & 11.283 & 1980.0 & -31 & 0 & 45 & 1980.00-1998.92 \\
\hline GDH & 69.252 & 306.467 & 1980.0 & 291 & -298 & 771 & 1980.00-1998.43 \\
\hline GLN & 49.650 & 262.883 & 1982.5 & $\cdots$ & $\cdots$ & $\cdots$ & $*$ \\
\hline GNA & -31.783 & 115.950 & 1980.0 & -35 & -143 & 103 & $1980.00-1998.83$ \\
\hline GUA & 13.560 & 144.870 & 1980.0 & 84 & 110 & 131 & 1980.00-1997.92 \\
\hline GVN & -70.667 & 351.733 & 1994.5 & $\cdots$ & $\cdots$ & $\cdots$ & $*$ \\
\hline $\mathrm{GZH}$ & 23.100 & 113.350 & 1980.0 & 52 & 96 & 33 & 1980.00-1997.92 \\
\hline HAD & 51.000 & 355.516 & 1980.0 & -64 & 24 & 88 & 1980.00-1998.92 \\
\hline HBK & -25.883 & 27.700 & 1980.0 & 27 & 27 & 32 & 1980.00-1998.75 \\
\hline HER & -34.416 & 19.233 & 1980.0 & -29 & 33 & -6 & 1980.00-1998.83 \\
\hline HIS & 80.617 & 58.050 & 1980.0 & 86 & -675 & 1121 & $*$ \\
\hline HLP & 54.600 & 18.817 & 1980.0 & 37 & -162 & -50 & 1980.00-1997.92 \\
\hline $\mathrm{HON}$ & 21.316 & 202.000 & 1980.0 & -189 & 64 & -312 & 1980.00-1997.92 \\
\hline HRB & 47.867 & 18.183 & 1980.0 & -5 & -34 & -20 & 1980.00-1998.92 \\
\hline HTY & 33.066 & 139.833 & 1981.5 & -34 & -794 & 458 & $*$ \\
\hline HYB & 17.417 & 78.550 & 1980.0 & 339 & 61 & 458 & $*$ \\
\hline IQA & 63.750 & 291.483 & 1995.5 & $\cdots$ & $\cdots$ & $\cdots$ & $*$ \\
\hline IRT & 52.166 & 104.450 & 1980.0 & 48 & 5 & -72 & 1980.00-1998.75 \\
\hline ISK & 41.067 & 29.067 & 1980.0 & 129 & 90 & -103 & 1980.00-1997.92 \\
\hline KAK & 36.233 & 140.183 & 1980.0 & -9 & 19 & -98 & 1980.00-1998.75 \\
\hline KIV & 50.716 & 30.300 & 1980.0 & 3 & 66 & 112 & 1980.00-1998.33 \\
\hline $\mathrm{KNY}$ & 31.416 & 130.883 & 1980.0 & -22 & 28 & -33 & 1980.00-1997.92 \\
\hline $\mathrm{KOU}$ & 5.217 & 307.267 & 1996.5 & $\cdots$ & $\cdots$ & $\cdots$ & $*$ \\
\hline $\mathrm{KRC}$ & 24.950 & 67.133 & 1985.5 & $\ldots$ & $\ldots$ & $\cdots$ & $*$ \\
\hline $\mathrm{KSH}$ & 39.500 & 76.000 & 1987.5 & $\cdots$ & $\cdots$ & $\cdots$ & $*$ \\
\hline LAS & -35.007 & 302.310 & 1980.0 & -8 & 1 & 33 & 1980.00-1997.17 \\
\hline LER & 60.133 & 358.816 & 1980.0 & -136 & 197 & 16 & 1980.00-1998.92 \\
\hline LNN & 59.950 & 30.700 & 1980.0 & 72 & 31 & -235 & $*$ \\
\hline LNP & 25.000 & 121.166 & 1980.0 & 3 & -16 & 133 & $*$ \\
\hline LOV & 59.350 & 17.833 & 1980.0 & 26 & 14 & 33 & $*$ \\
\hline LRV & 64.183 & 338.300 & 1980.0 & -256 & 594 & -503 & 1980.00-1997.92 \\
\hline LVV & 49.900 & 23.750 & 1980.0 & 152 & 103 & 165 & 1980.00-1997.92 \\
\hline LZH & 36.083 & 103.850 & 1980.0 & -18 & 9 & -84 & 1980.00-1997.92 \\
\hline MAB & 50.300 & 5.683 & 1991.0 & $\cdots$ & $\cdots$ & $\cdots$ & $*$ \\
\hline MAW & -67.600 & 62.883 & 1980.0 & -95 & 25 & -21 & $1980.00-1998.25$ \\
\hline $\mathrm{MBC}$ & 76.317 & 240.633 & 1980.0 & 34 & 10 & -121 & 1980.00-1997.42 \\
\hline MBO & 14.390 & 343.040 & 1980.0 & 80 & 47 & 160 & 1980.00-1998.92 \\
\hline MCQ & -54.500 & 158.950 & 1980.0 & 240 & 20 & 289 & $1980.00-1998.25$ \\
\hline MEA & 54.617 & 246.650 & 1980.0 & 69 & -4 & -100 & 1980.00-1997.92 \\
\hline MIR & -66.550 & 93.016 & 1980.0 & 68 & 380 & -197 & $*$ \\
\hline MIZ & 39.117 & 141.200 & 1980.0 & -88 & 52 & -269 & 1980.00-1998.83 \\
\hline MMB & 43.916 & 144.200 & 1980.0 & -253 & 149 & 72 & 1980.00-1997.92 \\
\hline MOL & -67.666 & 45.850 & 1980.0 & -84 & -223 & -415 & $*$ \\
\hline MOS & 55.466 & 37.316 & 1980.0 & 156 & -6 & 219 & 1980.00-1998.92 \\
\hline MZL & 49.600 & 117.400 & 1984.5 & -134 & 39 & -430 & $*$ \\
\hline NAQ & 61.160 & 314.560 & 1980.0 & -273 & 254 & 464 & 1983.00-1998.42 \\
\hline
\end{tabular}


Table 1. (continued).

\begin{tabular}{|c|c|c|c|c|c|c|c|}
\hline Code $^{a}$ & $\lambda^{b}$ & $\varphi^{c}$ & Date $^{d}$ & $X_{c c}(\mathrm{nT})^{e}$ & $Y_{c c}(\mathrm{nT})^{f}$ & $Z_{c c}(\mathrm{nT})^{g}$ & Time period ${ }^{h}$ \\
\hline NCK & 47.633 & 16.716 & 1980.0 & -14 & -12 & -21 & 1980.00-1998.92 \\
\hline NEW & 48.266 & 242.883 & 1980.0 & -70 & 91 & -47 & 1980.00-1997.92 \\
\hline NGK & 52.066 & 12.683 & 1980.0 & -49 & 10 & -41 & $1980.00-1998.83$ \\
\hline NUR & 60.516 & 24.650 & 1980.0 & 270 & -95 & 136 & $1980.00-1998.75$ \\
\hline NVS & 55.033 & 82.900 & 1980.0 & 151 & -134 & 94 & 1980.00-1998.92 \\
\hline ODE & 46.783 & 30.883 & 1980.0 & -101 & -735 & 21 & 1980.00-1998.33 \\
\hline OTT & 45.400 & 284.450 & 1980.0 & 122 & -169 & 122 & 1980.00-1997.92 \\
\hline OUL & 64.516 & 27.233 & 1993.5 & $\cdots$ & $\cdots$ & $\cdots$ & $*$ \\
\hline PAF & -49.350 & 70.200 & 1980.0 & 303 & -26 & -736 & 1980.00-1997.92 \\
\hline PBQ & 55.283 & 282.250 & 1980.0 & 127 & 360 & 34 & 1980.00-1997.92 \\
\hline PHU & 21.033 & 105.967 & 1996.5 & $\cdots$ & $\cdots$ & $\cdots$ & $*$ \\
\hline PPT & -17.567 & 210.417 & 1980.0 & -729 & -972 & 106 & 1980.00-1997.92 \\
\hline PST & -51.700 & 302.117 & 1994.5 & $\cdots$ & $\cdots$ & $\cdots$ & $*$ \\
\hline PTY & -17.250 & 292.050 & 1990.5 & $\cdots$ & $\cdots$ & $\cdots$ & $*$ \\
\hline QUE & 30.183 & 66.950 & 1980.0 & 62 & 89 & -8 & $*$ \\
\hline RES & 74.683 & 265.100 & 1980.0 & 54 & 49 & 28 & 1980.00-1997.92 \\
\hline SBA & -77.850 & 166.783 & 1980.0 & -2227 & -929 & -3766 & 1980.00-1998.92 \\
\hline SFS & 36.492 & 353.882 & 1991.5 & $\ldots$ & $\cdots$ & $\ldots$ & $*$ \\
\hline SIT & 57.066 & 224.683 & 1980.0 & -19 & 5 & -39 & 1980.00-1997.92 \\
\hline SJG & 18.116 & 293.850 & 1980.0 & -47 & 194 & 189 & 1980.00-1997.92 \\
\hline SOD & 67.366 & 26.633 & 1980.0 & -188 & -97 & -582 & 1980.00-1997.92 \\
\hline SPT & 39.550 & 355.650 & 1981.5 & 24 & 30 & -54 & 1980.00-1998.92 \\
\hline $\mathrm{SSH}$ & 31.100 & 121.183 & 1980.0 & -267 & 71 & 264 & 1980.00-1997.92 \\
\hline STJ & 47.600 & 303.317 & 1980.0 & 15 & 48 & -15 & 1980.00-1997.92 \\
\hline SUA & 44.683 & 26.250 & 1980.0 & -2 & -67 & -77 & 1980.00-1998.92 \\
\hline SYO & -69.000 & 39.583 & 1980.0 & -86 & -159 & -77 & $*$ \\
\hline SZT & 28.317 & 343.567 & 1980.0 & -3103 & -829 & -490 & $*$ \\
\hline TAM & 22.800 & 5.533 & 1980.0 & 24 & -232 & -39 & $*$ \\
\hline TAN & -18.916 & 47.552 & 1980.0 & 366 & -154 & -278 & $*$ \\
\hline TEO & 19.750 & 260.816 & 1980.0 & -25 & -95 & -39 & 1980.00-1998.92 \\
\hline TFS & 42.083 & 44.700 & 1980.0 & -269 & 51 & -134 & 1980.00-1998.75 \\
\hline THJ & 24.000 & 102.700 & 1985.5 & $\cdots$ & $\cdots$ & $\cdots$ & $*$ \\
\hline THL & 77.470 & 290.770 & 1980.0 & -55 & 148 & 23 & 1980.00-1998.42 \\
\hline THY & 46.900 & 17.900 & 1980.0 & -33 & -3 & -33 & 1980.00-1997.92 \\
\hline TIK & 71.583 & 129.000 & 1980.0 & -93 & -194 & -93 & $*$ \\
\hline TNG & -6.166 & 106.633 & 1980.0 & -1 & 5 & 70 & $*$ \\
\hline TRD & 8.483 & 76.950 & 1980.0 & 309 & 247 & 182 & 1980.00-1997.92 \\
\hline TRW & -43.267 & 294.617 & 1980.0 & 20 & 22 & 14 & 1980.00-1998.92 \\
\hline TSU & -19.200 & 17.583 & 1980.0 & 60 & -110 & 30 & 1980.00-1998.92 \\
\hline TTG & 3.516 & 98.566 & 1982.5 & 13 & 59 & -77 & $*$ \\
\hline TUC & 32.250 & 249.170 & 1980.0 & -112 & -325 & 440 & 1980.00-1997.92 \\
\hline VAL & 51.930 & 349.750 & 1980.0 & 75 & -42 & -7 & 1980.00-1998.92 \\
\hline VIC & 48.517 & 236.583 & 1980.0 & -22 & -8 & -195 & 1980.00-1997.92 \\
\hline VLA & 43.683 & 132.166 & 1980.0 & -22 & -20 & -53 & $*$ \\
\hline VOS & -78.450 & 106.866 & 1980.0 & -14 & 153 & -4 & $*$ \\
\hline VSS & -22.400 & 316.350 & 1980.0 & 60 & -96 & -14 & $*$ \\
\hline WHN & 30.533 & 114.566 & 1980.0 & 34 & 66 & -34 & 1980.00-1997.92 \\
\hline
\end{tabular}


Table 1. (continued).

\begin{tabular}{ccrccrcc}
\hline Code $^{a}$ & \multicolumn{1}{c}{$\lambda^{b}$} & \multicolumn{1}{c}{$\varphi^{c}$} & Date $^{d}$ & $X_{c c}(\mathrm{nT})^{e}$ & $Y_{c c}(\mathrm{nT})^{f}$ & $Z_{c c}(\mathrm{nT})^{g}$ & Time period $^{h}$ \\
\hline WMQ & 43.816 & 87.700 & 1980.0 & -42 & -98 & 146 & $*$ \\
WNG & 53.750 & 9.066 & 1980.0 & 32 & 67 & -47 & $1980.00-1997.92$ \\
YAK & 62.017 & 129.717 & 1980.0 & 69 & -1245 & 124 & $1980.00-1997.92$ \\
YKC & 62.483 & 245.517 & 1980.0 & -47 & -49 & -184 & $1980.00-1997.92$ \\
\hline
\end{tabular}

${ }^{a}$ According to the IAGA convention.

${ }^{b}$ Latitude of the observatory, in degrees.

${ }^{c}$ Longitude of the observatory, in degrees, positive eastward.

${ }^{d}$ Close on date to 1980 , used to estimate the crustal correction.

${ }^{e}$ Crustal contribution in $X$ component, in nT (geographic coordinates).

${ }^{f}$ Crustal contribution in $Y$ component, in nT (geographic coordinates)

${ }^{g}$ Crustal contribution in $Z$ component, in nT (geographic coordinates).

${ }^{h}$ Period over which monthly mean values were available (for SV modelling).

data were available. In the following we do not introduce an a priori model in our inversion procedure and we do not consider data already used in IGRF1995, such as POGS data or Project MAGNET data.

\section{Data}

Modelling of the main field and its secular variation is generally based on different sources of magnetic data. In the following we discuss two databases, one for the main field modelling, the other for the secular variation modelling. Also, we describe the applied interpolations and extrapolations.

\subsection{Data selection for main field modelling}

When deriving spherical harmonic models in the absence of MAGSAT-quality satellite data, it is important to collect data well distributed all over the globe and of good quality. Such models are therefore dependent on geomagnetic observatory data and data from repeat stations. Ship-borne magnetic survey measurements in areas without observatories were also used.

In our attempt to build a main field model for 2000.0 some data selection criteria were adopted. First, we decided to use only the magnetic data which were not included in modelling the main field for 1995 epoch (this gives us the possibility to compute a model which is independent of the IGRF1995). Then, we tried to obtain as many magnetic data as possible for the 1997.5 epoch. This epoch is the middle of the time-span between the two epochs for which IGRF models have to be available. It is also the last epoch for which secular variation models derived from observations rather than predictions can be computed-see Subsection 2.2.

2.1.1 Observatory data The present geographical distribution of observatories is still unbalanced between the Northern and the Southern hemispheres, and obviously between the continental and oceanic regions. Further deterioration is occurring because some observatories are closing, due to economic or political reasons. Also, obtaining the data for recent years is sometimes difficult, because some observatories have a long delay in computing and publishing their data. For the purpose of modelling, three field components were selected: northward $(X)$, eastward $(Y)$ and vertically downward $(Z)$. The list of available observatories with corresponding IAGA code and geographical coordinates is given in Table 1. The geographical distribution of these observatories is shown in Fig. 1. It is also to be noted that by using annual mean values the external field effects (diurnal variations, seasonal variations and magnetic disturbances) are mostly removed.

Two important problems have to be solved in preparing the final dataset of annual means. The first one is to get annual means for observatories which did not run throughout 1997. The second is to estimate the crustal biases.

Some observatories do not provide exact mean annual values for 1997.5 for various reasons: they were closed during that year, or they were run for a short while or they were closed before 1997 and new equipment was installed during that year. Two observatories were closed during 1997: GLN (annual mean from the first 85 days only, thereafter it was a variometer station), and $\mathrm{MBC}$ (observatory closed on day 174). In one observatory (AAE) new equipment was installed and data became available only from September 1997. To get the annual means the available data were extrapolated to 1997.5. In all, the data from 145 observatories were collected for this study.

The second important aspect relating to the preparation of the dataset is estimation of the crustal biases. It is known that some observatories are located on local anomalies that can exceed several hundred nanoTeslas (nT). To compute the crustal biases the annual mean value given by an observatory for the 1980.0 epoch was compared with the computed value (for the same location) from a model based on high quality vector data from the MAGSAT mission. We derived a model for 1980.0 using three models calculated from the quiet time data by Cohen (1989), i.e., 1979.871, 1980.027, 1980.120 epochs. Among the observatories listed in Table 1, only 114 observatories were operating during 1979-1980 (when changes in baselines occurred they have been applied). Three others changed their location since 1980 and the site corrections have not been supplied by the observatories, so we estimated these values (AAE, DIK, MIR). A few others were opened between 1980 and 1985, and we extrapolated data back to 1980 (AMS, CHD, CRP, CTA, DLR, ETT, FRN, 

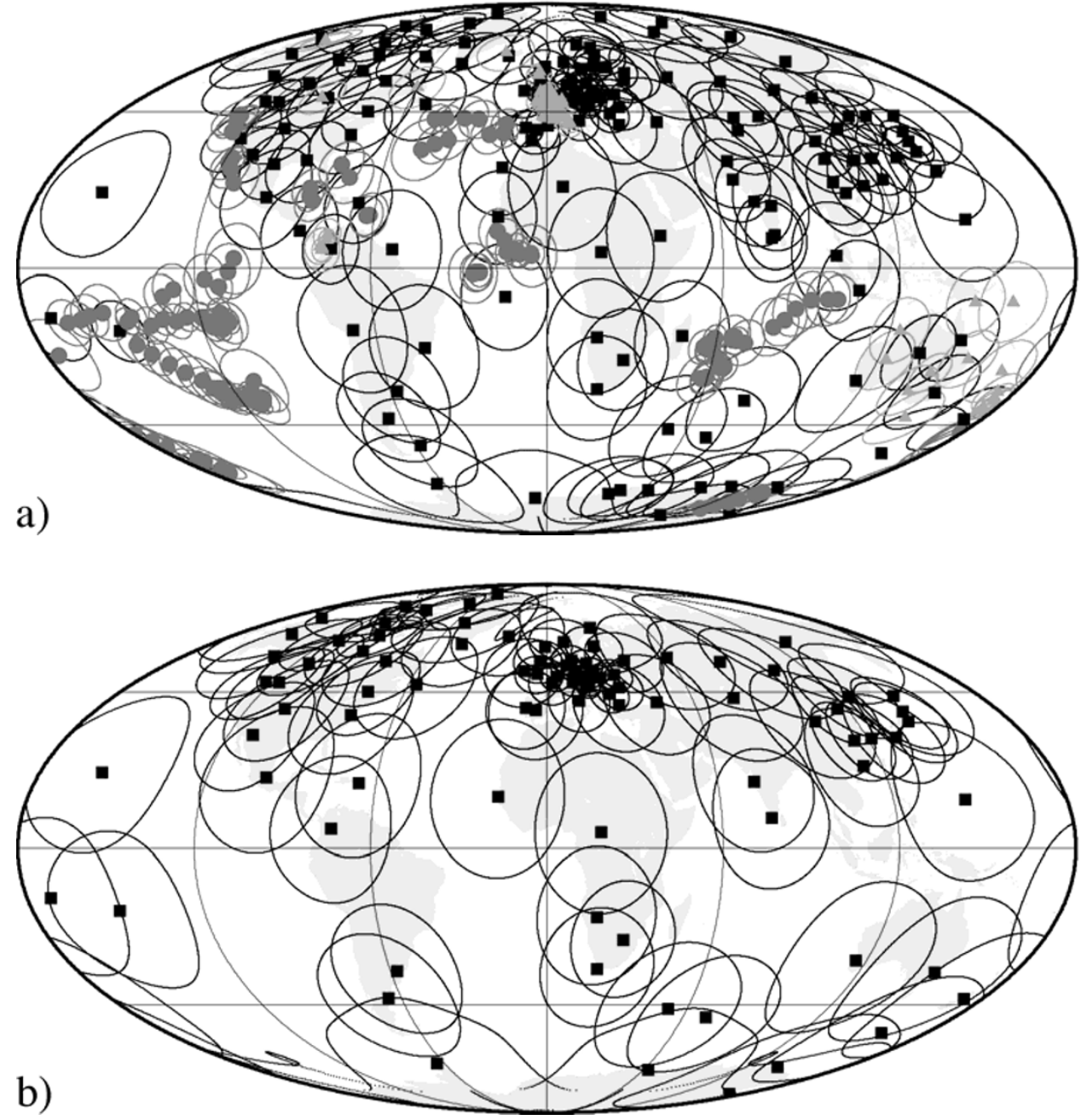

Fig. 1. a) Geographical distribution of the observations used in the main field modelling with their adopted weights: geomagnetic observatories (full black square), repeat stations (full pale grey triangle), scalar data (full grey circle). The weights are proportional here to the radius of circles around the observation points, and to a quality factor- 1 for observatories and $3 / 4$ for repeat stations and scalar data. b) Geographical distribution of the geomagnetic observatories used in the secular variation modelling with their adopted weights (proportional here to the radius of circles around the observation points). Mollweide equal area projection.

HTY, MZL, SPT and TTG). Also, the data for TAN were interpolated (linear interpolation).

For 16 observatories it was not possible to get mean values for 1980.0. We show later that these observatories are situated in areas without important magnetic anomalies, as indicated by the small magnitude of the absolute residuals with respect to our estimated 1997.5 model. Table 1 lists the crustal biases $\left(X_{c c}, Y_{c c}\right.$ and $\left.Z_{c c}\right)$, when computed.

The crustal biases, when available, were subtracted from each annual mean component. The final dataset was then converted to the geocentric coordinate system using the standard formulae (Langel, 1987).

2.1.2 Repeat station data Repeat stations are very useful in regional mapping. They are also very useful in global modelling, because of the reduced number of operating observatories in some large areas. The selected data were kindly provided by WDC Edinburgh, or directly by the national agencies. The repeat stations are generally reoccupied once every three to five years. For our purpose we retained only repeat stations occupied during 1997. Some of the available data were already reduced to the 1997.5 epoch.
The others were corrected assuming that the temporal change was the same as that at a nearby observatory.

All repeat station measurements were tested by comparison with our 1997.5 model (see Section 4), to eliminate those for which the absolute residuals from the model were greater than $250 \mathrm{nT}$ in any component. The final repeat station dataset contained 67 observations, distributed as shown in Fig. 1.

2.1.3 Scalar data Some marine surveys were made during 1995-1998. Marine data were selected from periods when $K_{p}$ indices were less than or equal to $2_{o}$, and only measurements made during local night time were used, to mitigate the effects of diurnal variations. No data with absolute residuals larger than $500 \mathrm{nT}$ with respect to our 1997.5 model were kept.

A map was then made of the selected data locations and from this it was decided that a $1^{\circ}$ by $1^{\circ}$ bin size was appropriate. For each bin a plot was made of total intensity versus time, and outliers were removed manually. The remaining data in the bin were averaged and adjusted to 1997.5 using the 1995.0-1999.0 secular variation models. 
The marine data were provided by: CITHER 3 LEG2 in the Atlantic Ocean (February-April 1995), LARJAKA in the Indian Ocean (October-November 1995), PRIMAR96 survey, in the Romanche-equatorial Atlantic area (May 1996), FUJI-MD108 survey, in the south-west Indian rift area (October-November 1997), FOUNDATION-HOT-LINE survey, in the south Pacific area (January-February 1997), MD112/MAGOFOND2 survey in the central Indian rift area (October-November 1998). Some other data were found on GEODAS vol. 4-"LEG" cruises. Six scalar measurements were provided by a polar expedition from Dumont D'Urville to Vostok (January-February 1996). In total there were 139 scalar data averages, the locations of which are also plotted in Fig. 1.

\subsection{Data selection for secular variation modelling}

Our aim is to obtain a series of annual secular variation, obtained by differencing main field models based on observatory annual means. To verify these annual mean data, and to extrapolate beyond the published values, we used observatory monthly means.

2.2.1 Monthly means from geomagnetic observatories In our view, the best method to map temporal changes on a time-scale of years or decades is to use data from geomagnetic observatories. It is emphasised that no survey or satellite data can provide such information. Furthermore, with using only satellite data, it is not possible to distinguish between temporal and spatial variations of the field; and the main field variations on temporal scales greater than years cannot be defined by occasional satellite missions, since such variations are not linear. The present-day distribution of observatories is far from ideal, and its effect on modelling has already been noted (Alexandrescu et al., 1994; Langel et al., 1995). In the following, we describe the observatory data used and the problems related to this dataset.

Our purpose was to obtain a dataset with as many observatories as possible containing long series of measurements. To ensure the consistency of data, and the stability of our secular variation model in time, we chose those observatories for which annual mean values cover the last two solar cycles (from 1975 to present).

Moreover, for modelling the secular variation, it is important to get a dataset of very good quality and to be able to extrapolate these data over a time-span of two-three years. These are the reasons we also used a database of monthly means (Alexandrescu, 1998). This database covers a shorter time-span than the database of annual means (for most observatories from 1980, until at least 1997-the end of the series depending on each observatory). All the monthly mean values were adjusted to the last known reference point, when changes were pointed out by the observatory.

Thereafter, using monthly mean series over more than 18 years, it is possible to simply plot the variations of each component and to see the consistency of the data. In this way we identified and corrected various mistakes, and we discovered some other jumps; when this situation was noted more information was directly requested from the observatories. The last problem to solve in preparing the series of monthly means was time gaps. In these cases, linear interpolation was used to reconstruct the missing values. The largest gaps we corrected for were six months long, with three exceptions
(AIA, API, and TSU). Longer gaps of about 2 years were exceptionally accepted, because they were all situated in the Southern hemisphere (but, only after confirmation from the observatories about the use of the same configuration before and after the interruption).

In all, the dataset of monthly mean values contains 93 observatories. They are listed in Table 1, together with the corresponding IAGA code, geographical coordinates, the beginning and the end of the time series. The geographical distribution of these observatories is shown in Fig. 1.

2.2.2 Extrapolation of monthly means The first use of monthly mean values was to verify the annual means: we computed the annual means from monthly means and compared them with the annual means published by WDCs. When the differences were relatively small $( \pm 5 \mathrm{nT})$ it was assumed they resulted from a different averaging procedure. However, for some observatories these differences were of the order of tens of nT. In all cases we asked for more information from the observatories, and we were able to find some sources of errors (sometimes wrong values tabulated in WDC files, sometimes different corrections which were not applied to monthly means, sometimes errors in initial computations).

The second use of monthly means was to extrapolate the available data over 2-3 years (depending on the end of each series) in order to reach 2000, the epoch of the IGRF candidate, and to cover the time-span from 1975 to 2000 for the secular variation models. To extrapolate the series we used exponential smoothing (Gardner, 1985). The specific formulae for simple exponential smoothing is:

$$
s_{t}=\alpha * x_{t}+(1-\alpha) * s_{t-1},
$$

where $s_{t}, s_{t-1}$ is the smooth signal, and $x_{t}$ the observed one. Consequently, each smoothed value is the weighted average of the previous observations, where the weights decrease exponentially depending on the value of parameter $\alpha$. If $\alpha$ is equal to 1 , then the previous observations are ignored entirely; if $\alpha$ tends to 0 , then the current observation is ignored entirely. Also we added a twelve-month seasonal variation, in agreement with the annual variation of the field. This seasonal component is derived in a similar way to the $s_{t}$ value from simple exponential smoothing. For this seasonal model we have:

$$
I_{t}=I_{t-p}+\delta *(1-\alpha) * e_{t}
$$

where $I_{t}$ is the seasonal component at time $t, I_{t-p}$ is the smoothed seasonal factor at time $t-p, p$ is the length of the period, and $e_{t}$ is an error, i.e., the difference between the observed and the forecast value at time $t$. If $\delta$ is 0 , a constant amplitude seasonal component is used to generate the onestep-ahead forecasts. If the $\delta$ parameter is equal to 1 , then the seasonal component is modified "maximally" at every step by the respective forecast error; in most of the series analyzed $\delta$ was zero.

All available $X, Y, Z$ components were fitted by this method, using a linear trend for most observatories (in some cases the exponential or damped trend had to be applied, when the series tendency was changing over the last part). All parameters were adjusted automatically, in a least squares sense. We show two examples of prediction, in Figs. 2 and 3. 

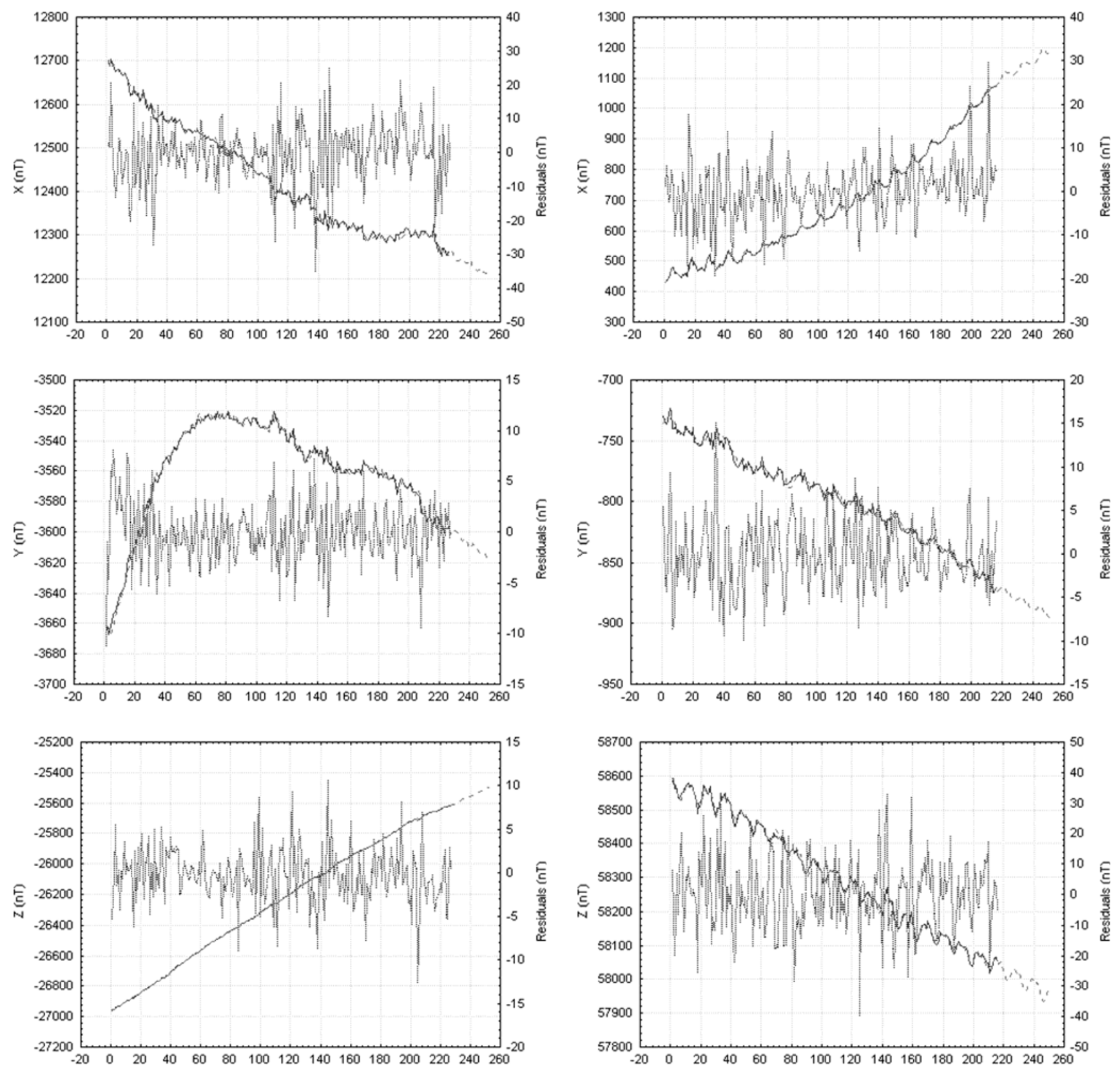

Fig. 2. Examples of measured series (full line), predicted values (dashed line) and residuals (dotted line) of the $X, Y, Z$ monthly means for Hartebeesthoek observatory (HBK), from 1998.75 to 2000.92 (time scale is indicated by the number of observations on the abscissa of each graph). The parameters used to extrapolate the $X, Y, Z$ components are $\alpha=0.539$, $0.675,0.727$ and $\gamma=0.0,0.118,0.045$ (see Subsection 2.2.2 for details).

\section{Method of Analysis}

For the main field model, epoch 1997.5 we used the dataset described in Subsection 2.1, and for the secular variation model 2000-2005 the datasets described in Subsection 2.2. All models were calculated using the least squares method of Cain et al. (1967). Also, we considered that the choice of the model for the first iteration has no importance in the final result, as it was shown recently (Ultré-Guérard, 1996). Considering the available data distribution, the model for the main field was computed up to degree and order 10, and the models for the secular variation up to degree and order 8 .

The weighting scheme applied to the geomagnetic obser-

Fig. 3. Examples of measured series (full line), predicted values (dashed line) and residuals (dotted line) of the $X, Y, Z$ monthly means for Resolute Bay observatory (RES), from 1998.75 to 2000.92 (time scale is indicated by the number of observations on the abscissa of each graph). The parameters used to extrapolate the $X, Y, Z$ components are $\alpha=0.720$, $0.408,0.507$ and $\gamma=0.0,0.007,0.007$ (see Subsection 2.2.2 for details).

vations is mainly based on the spatial distribution. Indeed, it is difficult to appreciate the quality of data provided by a given observation point because an important number of parameters which govern the field measurement cannot be parameterized (quality of instruments, operator experience, data processing, etc.).

The objective criterion in weighting data is to consider the geographical distribution of observation locations. For a given point, we search for all the other measurement positions situated in a circle of radius $(2 \cdot \pi \cdot a / 2 n)$, where $a(6371.2$ $\mathrm{km}$ ) denotes the mean radius of the Earth, and $n$ the maximum order of the spherical harmonic development; e.g., for the 

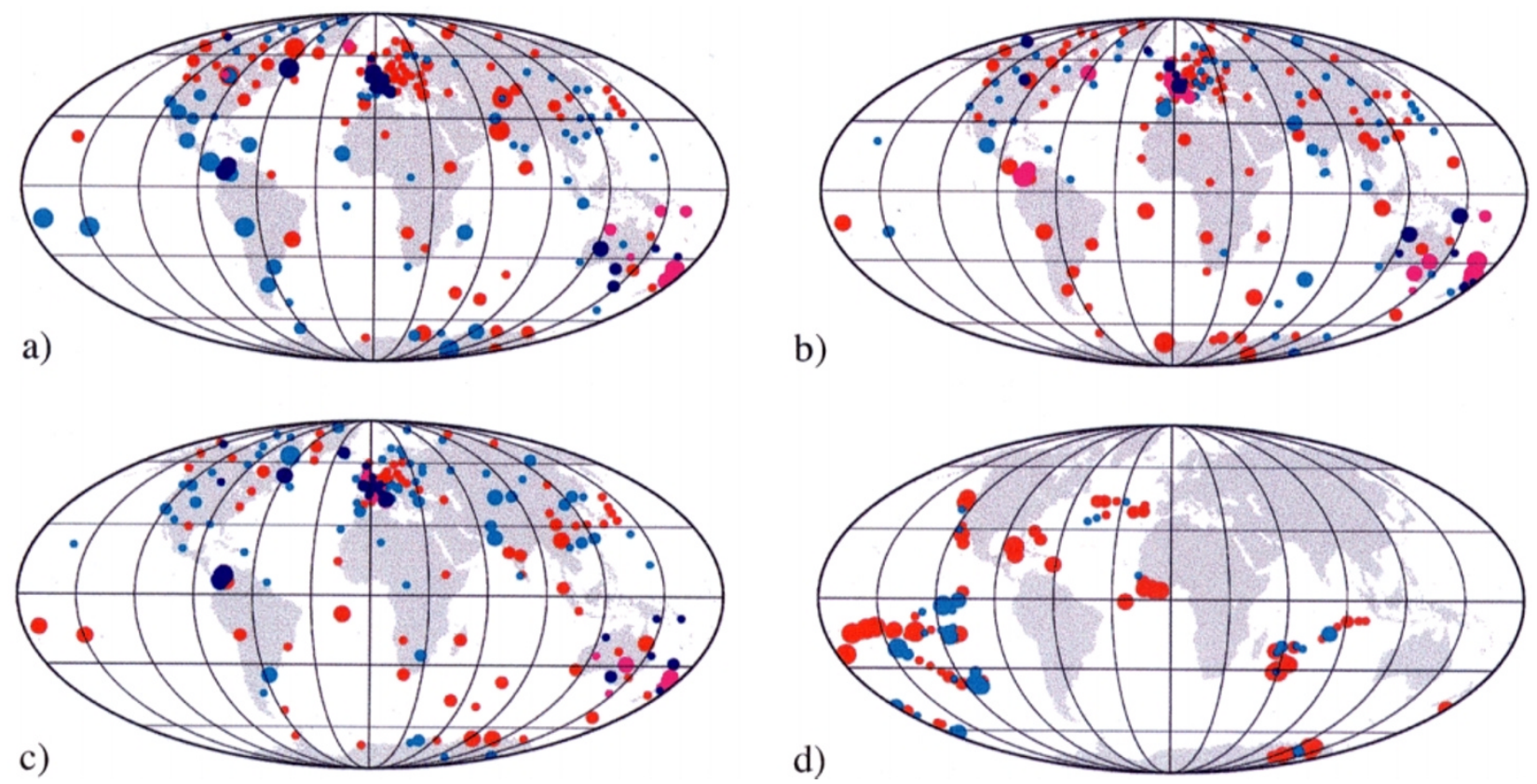

Fig. 4. Maps of residuals of the a) $X$, b) $Y$, c) $Z$, and d) $F$ components at the Earth's surface for the main field model at 1997.5 . The radius of circle are proportional to the absolute residuals: lower than $50 \mathrm{nT}$, between 50 and $100 \mathrm{nT}$, between 100 and $200 \mathrm{nT}$, and greater than $200 \mathrm{nT}$. Colors separate data sources and sign of residuals (negative/positive): observatory-light blue/red $(X, Y, Z)$, repeat stations-blue/purple $(X, Y, Z)$, scalar-light blue/red $(F)$. Mollweide equal area projection.

main field model developed up to degree and order 10 the radius is about $2000 \mathrm{~km}$. The circle represents the influence zone of the measurement on the model. This circle is divided into four quarters: south-east $(j=1)$, south-west $(j=2)$, north-west $(j=3)$ and north-east $(j=4)$ and then it can be written:

$$
\begin{gathered}
\omega_{i}=\frac{\omega_{i}^{\prime}}{T} \\
\omega_{i}^{\prime}=\frac{\sum_{j=1}^{4} \min \left(d_{j}, d_{\max }\right)}{4 \cdot d_{\max }} \cdot \gamma \\
T=\frac{\sum_{i=1}^{N} \omega_{i}^{\prime}}{N},
\end{gathered}
$$

where $\omega_{i}$ is the weight for the $i$ th observation, $d_{j}$ is the distance between the observation $i$ and the nearest one in the $j$ th quarter, $d_{\max }$ is the radius of the circle. The parameter $\gamma$ makes the distinction between different classes of observation, as a quality factor: here its value is set to 1 for each observatory and 3/4 for repeat stations and scalar data.

This weighting scheme did not give too large a weight to one isolated observation, and it did not eliminate the observations situated in areas with a good coverage (i.e., WesternEurope). Some other schemes, based also on minimal distance between the observations, were also developed and tested. In order to choose the most efficient one, we computed and plotted the covariance matrices of parameters. The method described above ensured the best diagonality of the computed covariance matrices. Figure 1 shows also an equivalent of the adopted weights, expressed in kilometers around a given location.

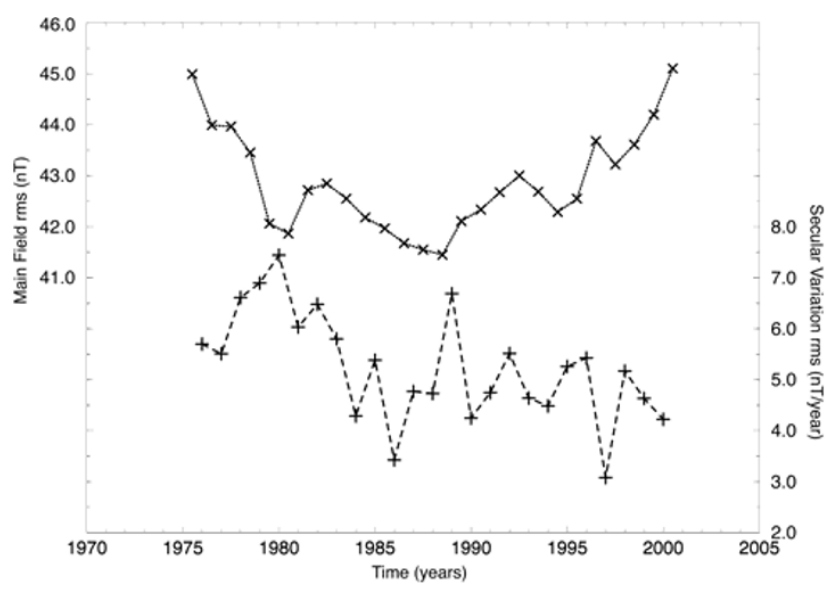

Fig. 5. Evolution of the root mean square residual (average over all components) for the MF (dotted line) and corresponding SV (dashed line) models from 1975 to 2000 .

\section{Results}

4.1 A proposed main field model for the 2000.0 epoch

The main field model for the 1997.5 epoch was computed up to degree and order 10. It is known that there are contributions beyond this limit, but the available data could not support a higher extension.

To extrapolate this main field model to the 2000.0 epoch we updated the 1997.5 model by using the annual secular variation models described below. The list of Gauss coefficients of the main field for the 2000.0 epoch is presented in Table 2. The 120 coefficients obtained were rounded to the nearest $\mathrm{nT}$.

The rms residual, average over all components, is $97 \mathrm{nT}$ 
Table 2. The Schimidt quasi-normalised main field coefficients $\left(g_{n}^{m}\right.$ and $\left.h_{n}^{m}\right)$ for 2000.0 and the secular variation ones $\left(g_{n}^{m}\right.$ and $\left.h_{n}^{m}\right)$ for $2000-2005$ time-span.

\begin{tabular}{|c|c|c|c|c|c|}
\hline Degree $n$ & Order $m$ & $g_{n}^{m}(\mathrm{nT})$ & $h_{n}^{m}(\mathrm{nT})$ & $g_{n}^{m}\left(\mathrm{nT} \mathrm{year}^{-1}\right)$ & $\dot{h_{n}^{m}}\left(\mathrm{nT}\right.$ year $\left.^{-1}\right)$ \\
\hline 1 & 0 & -29601 & $\ldots$ & 13.1 & $\ldots$ \\
\hline 1 & 1 & -1763 & 5173 & 9.3 & -25.0 \\
\hline 2 & 0 & -2284 & $\cdots$ & -12.2 & $\ldots$ \\
\hline 2 & 1 & 3095 & -2474 & -0.4 & -23.3 \\
\hline 2 & 2 & 1658 & -447 & -2.0 & -8.9 \\
\hline 3 & 0 & 1337 & $\ldots$ & 1.7 & $\cdots$ \\
\hline 3 & 1 & -2288 & -207 & -4.1 & 7.3 \\
\hline 3 & 2 & 1266 & 290 & 1.8 & 0.1 \\
\hline 3 & 3 & 749 & -471 & -8.1 & -14.9 \\
\hline 4 & 0 & 958 & $\ldots$ & -1.2 & $\cdots$ \\
\hline 4 & 1 & 775 & 263 & 2.4 & 2.8 \\
\hline 4 & 2 & 251 & -234 & -7.4 & 1.0 \\
\hline 4 & 3 & -416 & 121 & 4.1 & 6.4 \\
\hline 4 & 4 & 110 & -327 & -2.7 & -0.2 \\
\hline 5 & 0 & -221 & $\ldots$ & -0.6 & $\ldots$ \\
\hline 5 & 1 & 373 & 35 & -0.9 & -1.1 \\
\hline 5 & 2 & 207 & 188 & -3.0 & 0.2 \\
\hline 5 & 3 & -139 & -134 & -3.7 & 1.3 \\
\hline 5 & 4 & -158 & -52 & -0.7 & 2.9 \\
\hline 5 & 5 & -49 & 132 & 1.3 & 0.5 \\
\hline 6 & 0 & 74 & $\ldots$ & 0.9 & $\ldots$ \\
\hline 6 & 1 & 58 & -5 & -0.4 & -0.1 \\
\hline 6 & 2 & 68 & 34 & 0.3 & -0.9 \\
\hline 6 & 3 & -150 & 60 & 2.6 & 0.7 \\
\hline 6 & 4 & -16 & -49 & -0.6 & -0.4 \\
\hline 6 & 5 & 34 & -9 & 0.1 & -0.0 \\
\hline 6 & 6 & -65 & 43 & 0.9 & 1.2 \\
\hline 7 & 0 & 77 & $\ldots$ & 0.3 & $\ldots$ \\
\hline 7 & 1 & -103 & -65 & 0.2 & 1.6 \\
\hline 7 & 2 & 11 & -23 & 0.4 & -0.2 \\
\hline 7 & 3 & 41 & 19 & 1.4 & 0.3 \\
\hline 7 & 4 & 1 & 18 & 0.9 & 0.1 \\
\hline 7 & 5 & 7 & 27 & 0.5 & -1.4 \\
\hline 7 & 6 & 12 & -47 & 1.5 & -0.5 \\
\hline 7 & 7 & -9 & -13 & -1.4 & -0.2 \\
\hline 8 & 0 & 18 & $\ldots$ & -0.4 & $\ldots$ \\
\hline 8 & 1 & 18 & 5 & -0.1 & -0.6 \\
\hline 8 & 2 & -1 & -3 & -0.5 & 0.1 \\
\hline 8 & 3 & -19 & 9 & 0.0 & -0.2 \\
\hline 8 & 4 & -8 & -29 & -1.4 & -0.1 \\
\hline 8 & 5 & 16 & 9 & 0.0 & 1.0 \\
\hline 8 & 6 & 11 & 15 & -0.9 & -0.9 \\
\hline 8 & 7 & -14 & -6 & -0.6 & 0.6 \\
\hline 8 & 8 & -17 & 5 & -1.0 & -0.4 \\
\hline 9 & 0 & 4 & $\ldots$ & $\ldots$ & $\ldots$ \\
\hline 9 & 1 & 29 & -21 & $\ldots$ & $\ldots$ \\
\hline
\end{tabular}


Table 2. (continued)

\begin{tabular}{crrrcc}
\hline Degree $n$ & Order $m$ & $g_{n}^{m}(\mathrm{nT})$ & $h_{n}^{m}(\mathrm{nT})$ & $g_{n}^{m}\left(\mathrm{nT}\right.$ year $\left.^{-1}\right)$ & $h_{n}^{m}\left(\mathrm{nT} \mathrm{year}^{-1}\right)$ \\
\hline 9 & 2 & -1 & 11 & $\ldots$ & $\ldots$ \\
9 & 3 & -17 & 3 & $\ldots$ & $\ldots$ \\
9 & 4 & 3 & -8 & $\ldots$ & $\ldots$ \\
9 & 5 & -14 & 1 & $\ldots$ & $\ldots$ \\
9 & 6 & -11 & 11 & $\ldots$ & $\ldots$ \\
9 & 7 & 10 & 6 & $\ldots$ & $\ldots$ \\
9 & 8 & 3 & -28 & $\ldots$ & $\ldots$ \\
9 & 9 & -6 & 10 & $\ldots$ & $\ldots$ \\
10 & 0 & 0 & $\ldots$ & $\ldots$ & $\ldots$ \\
10 & 1 & -16 & 8 & $\ldots$ & $\ldots$ \\
10 & 2 & -2 & -10 & $\ldots$ & $\ldots$ \\
10 & 3 & 2 & 4 & $\ldots$ & $\ldots$ \\
10 & 4 & 3 & 9 & $\ldots$ & $\ldots$ \\
10 & 5 & 8 & -0 & $\ldots$ & $\ldots$ \\
10 & 6 & 2 & -11 & $\ldots$ & $\ldots$ \\
10 & 7 & 2 & -2 & $\ldots$ & $\ldots$ \\
10 & 8 & 9 & 10 & $\ldots$ & $\ldots$ \\
10 & 9 & -15 & 0 & $\ldots$ & $\ldots$ \\
10 & 10 & 6 & -3 & $\ldots$ & $\ldots$ \\
\hline
\end{tabular}

(respectively $101 \mathrm{nT}, 67 \mathrm{nT}$, and $117 \mathrm{nT}$ for observatory, repeat station, and scalar data). Figure 4 shows the residuals in $X, Y, Z, F$ components for each point of observation for the 1997.5 epoch. The mean values for the components are $-7 \mathrm{nT}, 14 \mathrm{nT}$, and $11 \mathrm{nT}$ in the observatories, $-16 \mathrm{nT}, 44$ $\mathrm{nT}$ and $3 \mathrm{nT}$ in the repeat stations, and $47 \mathrm{nT}$ for the scalar measurements.

\subsection{A proposed secular variation field for $2000-2005$}

To build the secular variation models over the 25 -year time-span, we computed 26 main field models, from 1975 to 1997 (for the middle of each year). These models have been used to estimate the secular variation models, by taking successive differences.

To verify the consistency of the datasets and of the results, the rms residuals for the main field and secular variation are plotted in Fig. 5. For the main field model, the mean of the rms residuals is $43 \mathrm{nT}$, and for the secular variation models it is $5 \mathrm{nT} \cdot \mathrm{yr}^{-1}$. As is shown in Fig. 5, the two curves may be correlated. The decrease of the rms residual for the main field models at the beginning of the period could be due to the improvement in the data quality (no monthly means were available between 1975 and 1980 to verify the annual mean values). On the contrary, the increase of the rms residual at the end of the interval is not explained by the data quality, as monthly means were available up to the end of 1998 (for almost all observatories).

From these models we extracted separately each coefficient. In such a way, series for each coefficient (i.e., series of $\dot{g}_{1}^{0}, \dot{g}_{1}^{1}, \dot{h}_{1}^{1}$, etc...) containing 25 values were produced.

To predict the time variation of these coefficients, we again used exponential smoothing. The prediction was not easy, because of the short length of the series. This was the reason we preferred to take constant value of $\alpha=0.5$ in the prediction procedure (Eq. (7)). Each series of coefficient was extrapolated to give five values, corresponding to the secular variation for 2001.0, 2002.0, 2003.0, 2004.0, and 2005.0. Finally, to predict a secular variation model for 2000-2005, we computed mean values for the 2002.5 epoch, as:

$$
\begin{aligned}
\dot{c}_{2002.5}= & {\left[\left(\frac{\dot{c}_{2000.0}+\dot{c}_{2005.0}}{2}\right)+\dot{c}_{2001.0}\right.} \\
& \left.+\dot{c}_{2002.0}+\dot{c}_{2003.0}+\dot{c}_{2004.0}\right] / 5
\end{aligned}
$$

where $\dot{c}$ represents a $\dot{g}_{n}^{m}$ or $\dot{h}_{n}^{m}$. The coefficients of this 2002.5 model are listed in Table 2, rounded to $0.1 \mathrm{nT} \cdot \mathrm{yr}^{-1}$.

\section{Conclusion}

In our attempt to produce a geomagnetic main field model for 2000.0 and a secular variation one for the 2000-2005 time-span, we chose an approach which makes no direct links between our models and previous IGRF models. It is pertinent to ask how valid these coefficients are. To answer this question a test was done, separately for the main field and secular variation.

\subsection{Evaluation of the proposed main field model}

We compared our main field model with the published IGRF 1995 model. The IGRF 1995 model was extended to 1997.5, using our secular variation models. Taking the difference between the two models, areas with important discrepancies have been noted. In the most densely covered areas the differences are of some tens of nT. As expected the worst situation is encountered in areas without observations (e.g., South Atlantic or North Pacific). But there were also 
large differences in areas where marine data were available for the main field model. For example, in the South Pacific the differences between the available marine data and those predicted by IGRF 1995 are about $1000 \mathrm{nT}$.

The large differences between our candidate model and the updated IGRF 1995 model show that the main geomagnetic field model has to be improved using the new Ørsted data. However at the time of these computation, data supplied by only the OverHauser magnetometer were available. Preliminary calculations using a part of this dataset showed that this improved our model. But, of course, vectorial data are really needed. With this new dataset a very good coverage of the Earth's surface will be realised.

\subsection{Evaluation of the proposed secular variation model}

We also tried to estimate the quality of our secular variation models, by checking the method of geomagnetic data prediction. For some observatories (chosen randomly), the last two years of monthly mean values were removed, and the same time-span was predicted. In all cases, predictions were in good agreement with data, except for the observatories where the secular variation rapidly changed.

The secular variation models would be improved if the data series were available from more observatories. In the present study, some observatories located in "key" areas like the Southern hemisphere are missing. However, the global network of geomagnetic observatories has a far from uniform distribution. So long as our knowledge of the secular variation depends heavily on observatory data, it will remain inaccurate. It is clear that new observatories are required, especially in ocean areas, and the long-term running of the observatories has also to be considered.

In the near future, new and exciting satellite missions (Ørsted, SAC-C, CHAMP) will investigate hitherto hidden features of the geomagnetic field. Only the combined analysis of data obtained by measurements at the Earth's surface and by satellites will drastically enhance the signal-to-noise ratio and extraction of the various sources of the field, en- abling a unique separation to be made.

Acknowledgments. The authors would like to express their thanks to the many observatories and agencies world-wide who forwarded their data. Other observatory data and repeat station data were provided by WDC A (Boulder) and WDC C1 (Edinburgh). We are extremely grateful to the following people who provided marine data: Michel Arhan, Enrico Bonatti, Jérôme Dyment, Yves Gallet, Marcia Maia, Catherine Mevel, Kensaku Tamaki, and Daniel Sauter. They also would like to thank Frank J. Lowes and two anonymous reviewers for their constructive comments. Special thanks to Jacques Bitterly, Susan Macmillan and Philippe Patriat for their help in obtaining some of the data. All maps have been plotted using the GMT software (Wessel and Smith, 1991). This is IPGP contribution 1704.

\section{References}

Alexandrescu, M., Database of geomagnetic observatory monthly means seeks contributors, EOS, 79, 345, 1998.

Alexandrescu, M., C. Ha Duyen, and J.-L. Le Mouël, Geographical distribution of magnetic observatories and field modelling, J. Geomag. Geoelectr., 46, 891-901, 1994.

Cain, J. C., S. J. Hendricks, R. A. Langel, and W. V. Hudson, A proposed model for the International Geomagnetic Reference Field-1965, J. Geomag. Geoelectr., 19, 335-355, 1967.

Cohen, Y., Traitements et interpretations de données spatiales en géomagnétisme: études des variations latérales d'aimantation de la lithosphère terrestre, Thèse, Université de Paris VII, 1989.

Gardner, E. S., Jr., Exponential smoothing: the state of the art, Journal of Forecasting, 4, 1-28, 1985.

Langel, R. A., Main field, in Geomagnetism, vol. 1, edited by J. A. Jacobs, pp. 249-512, Academic Press, London, 1987.

Langel, R. A., R. T. Baldwin, and A. W. Green, Toward an improved distribution of magnetic observatories for modelling of the main geomagnetic field and its temporal change, J. Geomag. Geoelectr., 47, 475-508, 1995.

Ultré-Guérard, P., Du paléomagnétisme au géomagnétisme spatial: analyse de quelques séquences temporelles du champ magnétique terrestre, Thèse, Institut de Physique du Globe de Paris, 1996.

Wessel, P. and W. H. F Smith, Free software helps map and display data, EOS Trans. Am. Geophys. Union, 72, 441-448, 1991.

B. Langlais (e-mail: langlais@ipgp.jussieu.fr) and M. Mandea (e-mail: mioara@ipgp.jussieu.fr) 